\title{
On Saab's Characterizations of Weak Radon-Nikodym Sets
}

\author{
By \\ Minoru MATSUDA*
}

\section{$\S 1$. Introduction}

Throughout this paper $X$ and $Y$ denote real Banach spaces with topological duals $X^{*}$ and $Y^{*}$ respectively. The closed unit ball in $X$ is denoted by $B_{X}$. Recently, in parallel with that of dual Banach spaces with the Radon-Nikodym property (RNP), the study of such spaces with the weak Radon-Nikodym property (WRNP) as well as Banach spaces not containing a copy of $l_{1}$ has been made by many authors, especially, Pelczynski [14], Rosenthal [18], [19], Odell and Rosenthal [13], Haydon [7], Musial [11], Janicka [9], Riddle and Uhr [17], and Saab and Saab [23]. Corresponding to those of dual Banach spaces with the RNP, a number of characterizations of such spaces with the WRNP have been obtained, heavily relying on Rosenthal's signal theorem (Theorem 1 in [18] or Theorem 2.2 in [19]) asserting that the space $X$ contains no copy of $l_{1}$ if and only if every bounded subset of $X$ is weakly pre-compact (For this terminology, see $\S 3$ ). They are collected below.

Theorem A. Each of the following statements characterizes $X$ not containing a copy of $l_{1}$.

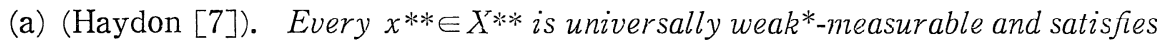
the barycentric formula (For this terminology, see $\S 2$ ) on $B_{X^{*}}$ equitped with the weak*-topology.

(b) (Pelczynski [14]). Every bounded linear operator $T: L_{1} \rightarrow X^{*}$ is a DunfordPettis operator.

(c) (Musial [11] and Janicka [9]). The space $X^{*}$ has the WRNP.

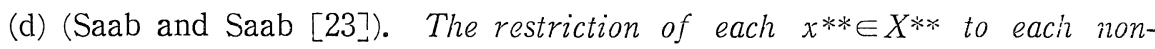
empty weak*-compact subset of $B_{X^{*}}$ has a point of weak*-continuity.

Succeedingly, as in the case of weak*-compact convex sets with the RNP (for instance, [20]), some attempts to localize the results stated in Theorem A

Communicated by S. Matsuura, December 24, 1984. Revised April 12, 1985.

* Department of Mathematics, Faculty of Science, Shizuoka University. Ohya. Shizuoka 422, Japan. 
have been made by Riddle, Saab and Uhr [16] and Saab [22]. They are stated as follows. First, by $[16]$,

Theorem B. If $K$ is a weak*-compact absolutely convex subset of $X^{*}$, then each of the following statements about $K$ implies all the others.

(a) Every $x^{* * \in X^{* *}}$ is universally weak*-measurable and satisfies the barycentric formula on $K$ equipped with the weak-topology.

(b) The set $K$ is a set of complete continuity (For this terminology, see $\S 3$ ).

(c) The set $K$ has the WRNP.

(d) The set $K$ has the scalar point of continuity property (For this terminology, see §4).

To show this, they proved the following Theorem $\mathrm{C}$, which forms the basis for most of [16].

Theorem C. Each of the following statements about a bounded linear operator $T: X \rightarrow Y$ implies all the others.

(a) The set $T\left(B_{X}\right)$ is weakly pre-compact.

(b) The operator $T$ factors through a Banach space containing no copy of $l_{1}$.

(c) The set $T *\left(B_{Y^{*}}\right)$ is a set of complete continuity.

(d) The set $T^{*}\left(B_{Y^{*}}\right)$ has the WRNP.

(e) The adjoint operator $T^{*}$ factors through a Banach space with the WRNP.

The proof of Theorem $\mathrm{C}$ depends on the factorization construction of Davis, Figiel, Johnson and Pelczynski [3] and Rosenthal's theorem stated above and it also depends on the following weak*-compact convex version of Janicka's result (cf. Theorem in [9]): If $K$ is a weak*-compact convex subset of $X^{*}$ such that every $x^{* *} \in X^{* *}$ is universally weak*-measurable and satisfies the barycentric formula on $K$ equipped with the weak*-topology, then $K$ has the WRNP, which was proved with the help of an important result (cf. Theorem 7.8.4 in [2]) arising from the theory of lifting (cf. [8]). Since this result also plays an essential role in our paper, we cite it as Theorem $\mathrm{I}$.

Theorem I. Let $(\Omega, \Sigma, \mu)$ be a complete finite measure space and let $K$ be a weak*-compact convex subset of $X^{*}$. If $\alpha: \Sigma \rightarrow X^{*}$ is a measure such that $\alpha(E) \in$ $\mu(E) \cdot K$ for each $E \in \Sigma$, then the following statements hold.

(a) There is an $f: \Omega \rightarrow K$ such that for each $x \in X$ the real-valued function $(f(\omega), x)$ is $\mu$-measurable and

$$
\int_{E}(f(\omega), x) d \mu(\omega)=(\alpha(E), x)
$$

for each $E \in \Sigma$ and $x \in X$.

(b) (A stronger form of (a)). There is a $g: \Omega \rightarrow K$ such that

(i) $g$ is $\Sigma-\mathfrak{B}(K)$ (the Borel $\sigma$-algebra in $K$ equipped with the weak*- 
topology) measurable.

(ii) $g(\mu)$ (the image measure of $\mu$ by $g$ ) is Radon on $K$ equipped with the weak*-topology.

(iii) $(\alpha(E), x)=\int_{E}(g(\omega), x) d \mu(\omega)$ for each $E \in \Sigma$ and $x \in X$.

Subsequently, making use of Theorem $2 \mathrm{~F}$ in [1] (A characterization theorem of point-wise relatively compact sets consisting of continuous functions in the space of universally measurable functions) and some results in [16] (for instance, Theorems B and C above), Saab [22] gave a following characterization theorem of weak*-compact convex sets with the WRNP, which is an extension of Theorems $\mathrm{A}$ and $\mathrm{B}$.

Theorem D. Let $K$ be a weak*-compact convex subset of $X^{*}$. Then the following statements about $K$ are equivalent.

(a) Every $x^{* *} \in X^{* *}$ is universally weak*-measurable on $K$ equitped with the weak*-topology.

(b) Every $x^{* *} \in X^{* *}$ is universally weak*-measurable and satisfies the barycentric formula on $K$ equipped with the weak*-topology.

(c) The set $K$ is a set of complete continuity.

(d) The set $K$ has the WRNP.

(e) The set $K$ has the scalar point of continuity property.

(f) Every bounded sequence $\left\{x_{n}\right\}_{n \geqq 1}$ in $X$ has a subsequence $\left\{x_{n(k)}\right\}_{k \geqq 1}$ such that for every $x^{*} \in K, \lim _{k \rightarrow \infty}\left(x^{*}, x_{n(k)}\right)$ exists.

As we have seen, Saab's characterization theorem of weak*-compact convex sets with the WRNP was verified through the factorization theorems (for instance, Theorem $\mathrm{C}$ above and Theorem 12 in [23]), the characterization theorem of weak*-compact absolutely convex sets with the WRNP and Theorem $2 \mathrm{~F}$ in [1]. Our first (and main) purpose of this paper is to give a slightly direct and vector-valued measure theoretic proof of the following Theorem E, a variant of Theorem D, without invoking any factorization theorem and absolutely convex argument.

Theorem E. Let $K$ be a weak*-compact convex subset of $X^{*}$. Then the following statements about $K$ are equivalent.

(a) Every $x^{* *} \in X^{* *}$ is universally weak*-measurable on $K$ equipsed with the weak*-topology.

(b) Every $x^{* *} \in X^{* *}$ is universally weak*-measurable and satisfies the barycentric formula on $K$ equipped with the weak-topology.

(c) The set $K$ is a set of complete continuity.

(d) The set $K$ is a set of complete continuity with respect to $([0,1], 1, \lambda)$ (For this terminology, see $\S 3$ ). 
(e) The set $K$ has the WRNP.

(f) The set $K$ has the WRNP with respect to $([0,1], \Lambda, \lambda)$.

(g) The set $K$ has the scalar point of continuity property.

(h) Every bounded sequence $\left\{x_{n}\right\}_{n \geqq 1}$ in $X$ has a subsequence $\left\{x_{n(k)}\right\}_{k \geqq 1}$ such that for every $x^{*} \in K, \lim _{k \rightarrow \infty}\left(x^{*}, x_{n(k)}\right)$ exists.

Here $([0,1], \Lambda, \lambda)$ denotes the Lebesgue measure space on $[0,1]$. Now the most interesting (and different) point of our proof is that we make the best use of the following Theorem 3.17 in [19] and the well-known lifting theorem for vector measures rather than Theorem 2.2 in [19] and factorization theorems. Furthermore it should be noted that each of Rosenthal's Theorems 2.2 and 3.17 in [19] can be proved by the almost same process and the lifting theorem for vector measures can be proved simply by a Lindenstrauss compactness argument.

Theorem II (Theorem 3.17 in [19]). Let $Z$ be a Polish space and $\left(A_{n}, B_{n}\right)_{n \geqq 1}$ a sequence of pairs of subsets of $Z$ with $A_{n}, B_{n}$ closed and $A_{n} \cap B_{n}=\phi$. Assume that $\left(A_{n}, B_{n}\right)_{n \geqq 1}$ has no convergent subsequence (For this terminology, see §3). Then there exist a compact subset $\Gamma$ of $Z$ homeomorphic to $\Delta\left(=\{0,1\}^{N}\right.$, the Cantor set), a homeomorphism $\sigma$ from $\Gamma$ onto $\Delta$, and a sequence $n(1)<n(2)<\cdots$ such that $A_{n(k)} \cap \Gamma=\sigma^{-1}\left(U_{k}\right)$ and $B_{n(k)} \cap \Gamma=\sigma^{-1}\left(U_{k}^{c}\right)$ for all $k$. Here $U_{k}=\left\{s=\left\{s_{n}\right\}_{n \geqq 1} \in\right.$ $\left.\Delta: s_{k}=0\right\}$.

Theorem III. Let $T: X \rightarrow Y$ be a bounded linear operator and let $(\Omega, \Sigma, \mu)$ be a finite measure space. If $K$ is a weak*-compact convex subset of $Y^{*}$, then for every measure $\alpha: \Sigma \rightarrow X^{*}$ such that $\alpha(E) \in \mu(E) \cdot T^{*}(K)$ for each $E \in \Sigma$, there exists a measure $\tau: \Sigma \rightarrow Y^{*}$ such that

(1) $\tau(E) \in \mu(E) \cdot K$ for each $E \in \Sigma$,

(2) $T * \tau(E)=\alpha(E)$ for each $E \in \Sigma$.

The combination of Theorems I, II and III makes us possible to study the properties of weak*-compact convex sets with the WRNP and to give a proof of Theorem $\mathrm{E}$ in a way (that is, a way parallel with one in the case of Theorem A) different from Riddle, Saab and Uhr [16] and Saab [22]. This may allow us to say that Theorems I, II and III are fundamental on the study of weak*compact convex sets with the WRNP. Moreover, seeing that a lifting theorem for vector measures of finite variation is applicable to give some characterizations of $B_{X^{*}}$ with the WRNP (cf. [9] and [12]), we think it meaningful to make the best use of the lifting theorem for vector measures (Theorem III) which is considered to be convenient for the study of weak*-compact convex sets with the WRNP.

In $\S 2$, making use of Theorem III, we reconsider the relation among the statements (a), (b) and (e) in Theorem $\mathrm{E}$ by the medium of $l_{1}$ and establish their equivalence. At this time, we also use Haydon's characterization of bary- 
centric calculus (Proposition 4.1 in [7]). This gives us a more direct and concrete proof of the equivalence among these statements. Furthermore, suggested by this proof, we can give a localization of a corresponding result concerning the WRNP of dual Banach spaces (cf. Theorem $5^{\prime}$ in [11]) making use of Theorems I, II and III, which is the second purpose of this paper. In $\S 3$, we first introduce the notion of relative weakly pre-compact sets. Concerning this, making use of Theorems I, II and III, we give an analogue (that is, a localized one) of Theorem 1 in [16], which is the third purpose of this paper. We also establish the equivalence among the statements (c), (d), (e), (f) and (h) in Theorem E making use of Theorems I, II and III. It should be noted that in connection with Theorems II and III the negative statement of (h) in Theorem E is very essential and Theorem III is very effective on this proof. Furthermore, the equivalence among these statements yields a localization of some parts (that is, the statements (a), (c) and (d)) of Theorem $\mathrm{C}$ and some corollaries. Finally, in $\S 4$, applying one of the corollaries and a result in the case where $X$ is separable, we see that the statements (e) and (g) in Theorem E are equivalent.

\section{$\S 2$. Universal Measurability, Barycentric Calculus and the Weak Radon-Nikodym Property}

In what follows, we always understand that for every weak*-compact convex subset $K$ of a dual Banach space $X^{*}, K$ is equipped with the weak*-topology. If $S$ is a compact Hausdorff space, then $\mathfrak{B}(S)$ denotes the Borel $\sigma$-algebra in $S$. A real-valued function $k$ defined on $S$ is said to be universally measurable if $k$ is $\sigma$-measurable for every Radon probability measure $\sigma$ on $S$; that is, by Lusin's theorem, if and only if there exists for each such measure $\sigma$ and $\varepsilon>0$ a compact $F \subset S$ with $\sigma(S \backslash F)<\varepsilon$ and $k_{\mid F}$ (the restriction of $k$ to $F$ ) continuous. In the case where $K$ is a weak*-compact convex subset of $X^{*}$, we say that a universally measurable affine function $f$ on $K$ satisfies the barycentric formula if we have

$$
f(b(\sigma))=\int_{K} f\left(x^{*}\right) d \sigma\left(x^{*}\right)
$$

for any Radon probability measure $\sigma$ on $K$. Here $b(\sigma)$ denotes the barycenter of $\sigma$, defined to be the unique point of $K$ such that

$$
h(b(\sigma))=\int_{K} h(x *) d \sigma(x *)
$$

for every continuous affine function $h$ on $K$. Following Haydon [7], we also say that $f$ satisfies the barycentric calculus on $K$ if $f$ is universally measurable and satisfies the barycentric formula on $K$. So, for instance, the statement (b) in Theorem $\mathrm{E}$ shows that every $x^{* *} \in X^{* *}$ satisfies the barycentric calculus on $K$.

In the following, $(\Omega, \Sigma, \mu)$ always denotes a complete finite measure space. For each $(\Omega, \Sigma, \mu)$, a function $f: \Omega \rightarrow X^{*}$ is said to be weak*-measurable if for 
each $x \in X$ the real-valued function $(f(\omega), x)$ is $\mu$-measurable. Two weak*measurable function $f: \Omega \rightarrow X^{*}$ and $g: \Omega \rightarrow X^{*}$ are called weak* equivalent provided, for every $x \in X,(f(\boldsymbol{\omega}), x)=(g(\boldsymbol{\omega}), x) \mu$-a. e. We say that a function $f: \Omega \rightarrow X$ is weakly measurable if for each $x^{*} \in X^{*}$ the real-valued function $\left(x^{*}, f(\omega)\right)$ is $\mu$ measurable. A weakly measurable function $f: \Omega \rightarrow X$ is said to be Pettis integrable if $\left(x^{*}, f(\omega)\right) \in L_{1}(\Omega, \Sigma, \mu)$ for evey $x^{*} \in X^{*}$ and moreover for each $E \in \Sigma$ there exists an element $x_{E} \in X$ that satisfies

$$
\left(x^{*}, x_{E}\right)=\int_{E}\left(x^{*}, f(\omega)\right) d \mu(\omega)
$$

for every $x^{*} \in X^{*}$.

A closed, bounded and convex subset $C$ of $X$ is said to have the WRNP with respect to $(\Omega, \Sigma, \mu)$ if for every measure $\alpha: \Sigma \rightarrow X$ such that $\alpha(E) \in \mu(E) \cdot C$ for every $E \in \Sigma$, there exists a Pettis integrable function $g: \Omega \rightarrow C$ such that

$$
\left(x^{*}, \alpha(E)\right)=\int_{E}\left(x^{*}, g(\omega)\right) d \mu(\omega)
$$

for each $E \in \Sigma$ and $x * \in X^{*}$. We say that the set $C$ has the WRNP if $C$ has this property with respect to every $(\Omega, \Sigma, \mu)$. Such a set $C$ is called a weak Radon-Nikodym set. A Banach space $X$ is said to have the WRNP (resp. WRNP with respect to $(\Omega, \Sigma, \mu)$ ) if $B_{X}$ is a weak Radon-Nikodym set (resp. $B_{X}$ has the WRNP with respect to $(\Omega, \Sigma, \mu)$ ).

In this section we want to clarify the equivalence among the statements (a), (b) and (e) in Theorem $\mathrm{E}$ by the medium of $l_{1}$. (In fact, seeing that the equivalence between the statements (a) and (c) in Theorem A became clear through the good offices of $l_{1}$, it is natural for us to wish to find out a statement about $K$ in terms of $l_{1}$ which acts as intermediary in this case.) To this end, let us recall some basic facts on $l_{\infty}^{*}$, the double dual of $l_{1}$. We first note that $l_{\infty}^{*}$ may be regarded as the Banach space of all bounded finitely additive measure on $\mathscr{L}(N)$, the set of all subsets of $N$. From this we know that there is a one-to-one correspondence between multiplicative bounded linear functionals on $l_{\infty}$ and finitely additive zero-one measures on $\mathscr{L}(N)$. Furthermore we see that the set of finitely additive zero-one measures is in one-to-one correspondence with the set of all ultrafilters on $N$ by assigning to an ultrafilter $\mathscr{F}$ on $N$ the set function $\eta$ defined for $A \in \mathscr{L}(N)$ by putting $\eta(A)=1$ or 0 according as $A \in \mathscr{F}$ or $A \notin \mathscr{F}$. Hence there is a one-to-one correspondence between multiplicative bounded linear functionals on $l_{\infty}$ and ultrafilers on $N$. We denote by $L_{\Phi}$ the corresponding element of $l_{\infty}^{*}$ to $\mathscr{I}$ for each ultrafilter $\mathscr{I}$ on $N$.

Consider the following statement $(*)$ about $K$ which acts as intermediary.

(*) For every bounded linear operator $T: l_{1} \rightarrow X$ and every nonprincipal ultrafilter $\mathbb{I}$ on $N, L_{q}$ is universally measurable on $T *(K)$.

Then we have: 
Proposition 1. Let $K$ be a weak*-compact convex subset of $X^{*}$. Then the following statements about $K$ are equivalent.

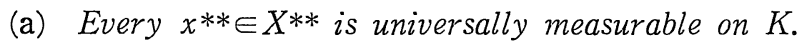

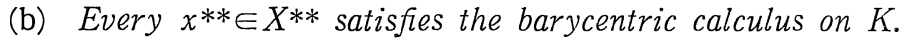

(c) The statement (*) holds.

(d) The set $K$ has the WRNP.

Before proving Proposition 1, we need the

Definition 1 ([18]). A sequence $\left(A_{n}, B_{n}\right)_{n \geqq 1}$ of pairs of subsets of some set is called independent provided $A_{n} \cap B_{n}=\phi$ for all $n$ and for any two disjoint finite subsets $N_{1}$ and $N_{2}$ of $N$

$$
\bigcap_{n \in N_{1}} A_{n} \cap \bigcap_{n \in N_{2}} B_{n} \neq \dot{\phi}
$$

That is, for all $\left\{\varepsilon_{n}\right\}_{n=1}^{k}$ with $\varepsilon_{n}= \pm 1, \bigcap_{n=1}^{k} \varepsilon_{n} A_{n} \neq \phi$ where $\varepsilon_{n} A_{n}=A_{n}$ if $\varepsilon_{n}=+1$ and $\varepsilon_{n} A_{n}=B_{n}$ if $\varepsilon_{n}=-1$.

Haydon [7] showed that if $X$ contains no copy of $l_{1}$, then every $x * * \in X^{* *}$ satisfies the barycentric calculus on $B_{X^{*}}$. Replacing $B_{X^{*}}$ by a weak*-compact convex subset $K$ of $X^{*}$ in Haydon's proof yields the following:

Lemma 1. Let $K$ be a weak*-compact convex subset of $X^{*}$. If there exists an $x^{* *} \in X^{* *}$ which does not satisfy the barycentric calculus on $K$, then there exist a non-empty compact subset $S$ of $K$, real numbers $r$ and $\delta$ with $\delta>0$ and $a$ bounded sequence $\left\{x_{n}\right\}_{n \geq 1}$ in $X$ such that $\left(A_{n}, B_{n}\right)_{n \geqq 1}$ is independent, where $A_{n}=\{x * \in S$ : $\left.\left(x *, x_{n}\right) \geqq r+\delta\right\}$ and $B_{n}=\left\{x * \in S:\left(x^{*}, x_{n}\right) \leqq r\right\}$.

We are now in a position to prove Proposition 1.

Proof of Proposition 1. ( $\alpha$ ) First let us prove that (c) implies (b) and that (a) implies (b). Our proof of this part depends on the argument employed in the proof of (i) $\rightarrow$ (vi) in Theorem $2 \mathrm{~F}$ of [1]. Suppose that (b) fails. Then we have a non-empty compact subset $S$ of $K$, real numbers $r$ and $\delta$ with $\delta>0$ and a bounded sequence $\left\{x_{n}\right\}_{n \geq 1}$ in $X$ as stated in Lemma 1. Define a bounded linear operator $T: l_{1} \rightarrow X$ by $T\left(\left\{a_{n}\right\}_{n \geqq 1}\right)=\sum_{n=1}^{\infty} a_{n} x_{n}$ for every $\left\{a_{n}\right\}_{n \geqq 1} \in l_{1}$. Especially, $T\left(e_{n}\right)=x_{n}$ for all $n$. Here $e_{n}$ denotes the $n$-th unit vector of $l_{1}$. Further $T * x^{*}$ $=\left\{\left(x^{*}, x_{n}\right)\right\}_{n \geqq 1} \in l_{\infty}$ for every $x^{*} \in X^{*}$. Let $A_{n}$ and $B_{n}$ be the same as in Lemma 1. Then $\bigcap_{n=1}^{\infty} \varepsilon_{n} A_{n} \neq \phi$ for all $\left\{\varepsilon_{n}\right\}_{n \geqq 1}$ with $\varepsilon_{n}= \pm 1$, since $\left(A_{n}, B_{n}\right)_{n \geqq 1}$ is independent and $S$ is compact. Putting $C_{n}=T *\left(A_{n}\right)\left(=\left\{a * \in T *(S):\left(a *, e_{n}\right) \geqq r+\delta\right\}\right)$, and $D_{n}$ $=T^{*}\left(B_{n}\right)\left(=\left\{a^{*} \in T^{*}(S):\left(a^{*}, e_{n}\right) \leqq r\right\}\right)$, it is easily seen that $\left(C_{n}, D_{n}\right)_{n \geqq 1}$ is in- 
dependent and so for all $\left\{\varepsilon_{n}\right\}_{n \geq 1}$ with $\varepsilon_{n}= \pm 1, \bigcap_{n=1}^{\infty} \varepsilon_{n} C_{n} \neq \phi$, where $\varepsilon_{n} C_{n}=C_{n}$ if $\varepsilon_{n}=+1$ and $\varepsilon_{n} C_{n}=D_{n}$ if $\varepsilon_{n}=-1$. Set $E=\left\{a^{*} \in T^{*}(S):\left(a^{*}, e_{n}\right) \geqq r+\delta\right.$ or $\left(a^{*}, e_{n}\right)$ $\leqq r$ for any $n \in N\} \quad\left(=\bigcap_{n=1}^{\infty}\left(C_{n} \cup D_{n}\right)\right)$. Then $E$ is a non-empty compact subset of $T *(K)$. Define $\phi: E \rightarrow \mathscr{Q}(N)$ ( $:$ regarded as the Cantor set with its usual compact metric topology and its usual measure, that is, normalized Haar measure if we identify $\mathscr{P}(N)$ with $\{0,1\}^{N}$ ) by writing

$$
\phi(a *)=\left\{n:\left(a^{*}, e_{n}\right) \geqq r+\delta\right\}
$$

for every $a * \in E$. Then $\phi$ is a continuous surjection. Let $\nu$ be the normalized Haar measure on $\mathscr{Q}(N)$. Then, by a well-known theorem (see Theorem 2.2 in [5]), we have a Radon probability measure $\mu$ on $E$ such that $\nu=\phi(\mu)$. Let $L \in l_{\infty}^{*}$ be any weak*-cluster point of a sequence $\left\{e_{n}\right\}_{n \geqq 1}$ in $l_{\infty}^{*}$. Then there exists a nonprincipal ultrafilter $\mathscr{F}$ on $N$ such that $L=L_{\mathscr{F}}$. Furthermore $\lim _{n \rightarrow \mathscr{G}}\left(a^{*}, e_{n}\right)$ $=L_{q}\left(a^{*}\right)$ for every $a^{*} \in E$. Hence we see that for $a^{*} \in E$,

$$
L_{\mathcal{F}}\left(a^{*}\right) \geqq r+\delta \longleftrightarrow\left\{n:\left(a^{*}, e_{n}\right) \geqq r+\delta\right\} \in \mathscr{F} \longleftrightarrow \phi\left(a^{*}\right) \in \mathscr{I} .
$$

So $\phi^{-1}(\mathscr{I})=\left\{a^{*} \in E: L_{\mathscr{I}}\left(a^{*}\right) \geqq r+\delta\right\}$. But $\mathscr{I}$ is not measurable for $\nu$ by Lemma $2 \mathrm{D}$ in [1] and so $\phi^{-1}(\mathscr{F})$ can not be measurable for $\mu$ (see [24], Chapter $1, \S 1$, Theorem 9, p. 35). Consequently, $L_{q}$ is not measurable for $\bar{\mu}$ where $\bar{\mu}$ is the Radon probability measure on $T^{*}(K)$ extending $\mu$. Thus $L_{\mathscr{T}}$ is not universally measurable on $T *(K)$ and so (c) fails. Moreover, we know the existence of a Radon probability measure $\theta$ on $K$ satisfying $T_{r}^{*}(\theta)=\bar{\mu}$, where $T_{r}^{*}$ is the restriction of $T^{*}$ to $K$. Then $T^{* *} L_{\Phi}$ is not measurable for $\theta$, since $L_{\Phi}$ is not measurable for $\bar{\mu}$. Thus $x^{* *}=T^{* *} L_{\Phi} \in X^{* *}$ is not universally measurable on $K$. So (a) fails.

( $\beta$ ) Next let us prove that (d) implies (c) making use of Theorem III. This is the part to be emphasized in our proof. Suppose that (c) fails. Then there exist a bounded linear operator $T: l_{1} \rightarrow X$ and an element $L_{\mathscr{F}}$ of $l_{\infty}^{*}$ such that $L_{\mathscr{F}}$ is not universally measurable on $T^{*}(K)$. Let $\gamma$ be a Radon probability measure on $T *(K)$ such that $L_{\Phi}$ is not measurable for $\gamma$. Consider the complete finite measure space $\left(T *(K), \mathfrak{M}_{\gamma}, \gamma\right)$, where $\mathfrak{M}_{\gamma}$ denotes the set of all $\gamma$-measurable subsets of $T^{*}(K)$. Define $\alpha: \mathfrak{M}_{\gamma} \rightarrow l_{\infty}$ by $\alpha(E)=0$ if $\gamma(E)=0$ and $\alpha(E)=\gamma(E) \cdot b\left(\gamma_{E}\right)$ if $\gamma(E)>0$, where $\gamma_{E}$ denotes the Radon probability measure on $T *(K)$ defined by $\gamma_{E}(B)=\gamma(E \cap B) / \gamma(E)$ for every $B \in \mathfrak{B}\left(T^{*}(K)\right)$. Then $\alpha$ is an $l_{\infty}$-valued vector measure that satisfies $\alpha(E) \in \gamma(E) \cdot T *(K)$ for each $E \in \mathfrak{M}_{\gamma}$. So, in virtue of Theorem III, there exists a measure $\beta: \mathfrak{M}_{\gamma} \rightarrow X^{*}$ such that

(1) $\beta(E) \in \gamma(E) \cdot K$ for each $E \in \mathfrak{M}_{\gamma}$,

(2) $T * \beta(E)=\alpha(E)$ for each $E \in \mathfrak{M}_{\gamma}$.

Suppose that $K$ has the WRNP, then by (1), there exists a Pettis integrable function $g: T *(K) \rightarrow K$ such that 


$$
\left(x^{* *}, \beta(E)\right)=\int_{E}\left(x^{* *}, g\left(a^{*}\right)\right) d \gamma\left(a^{*}\right)
$$

for each $E \in \mathfrak{M}_{\gamma}$ and $x^{* *} \in X^{* *}$. Now we consider the $l_{\infty}$-valued weakly measurable function $h\left(a^{*}\right)=T^{*} g\left(a^{*}\right)$ on $T^{*}(K)$. Since $T^{* *} L \in X^{* *}$ for "every $L \in l_{\infty}^{*}$, we have

$$
(T * * L, \beta(E))=\int_{E}(T * * L, g(a *)) d \gamma(a *)
$$

for each $E \in \mathfrak{M}$, and so

$$
(L, T * \beta(E))=\int_{E}(L, T * g(a *)) d \gamma(a *) .
$$

Hence, for every $L \in l_{\infty}^{*}$, we have by (2),

$$
(L, \alpha(E))=\int_{E}\left(L, h\left(a^{*}\right)\right) d \gamma\left(a^{*}\right)
$$

for each $E \in \mathfrak{M}_{\gamma}$. In particular, we have

$$
(\alpha(E), a)=\int_{E}\left(h\left(a^{*}\right), a\right) d \gamma\left(a^{*}\right)
$$

for each $E \in \mathfrak{M}_{\gamma}$ and $a \in l_{1}$. By the way, it follows from the definition of $\alpha$ that

$$
(\alpha(E), a)=\int_{E}\left(a^{*}, a\right) d \gamma\left(a^{*}\right)
$$

for each $E \in \mathfrak{M}_{\gamma}$ and $a \in l_{1}$. Thus, by the use of the separability of $l_{1}$, we can deduce that $h\left(a^{*}\right)=a^{*} \gamma$-a. e. and so $\left(L_{q}, h\left(a^{*}\right)\right)=L_{q}\left(a^{*}\right) \gamma$-a. e. So $\left(L_{q}, h\left(a^{*}\right)\right)$ is not measurable for $\gamma$, which contradicts that $h$ is weakly measurable. Hence we complete the proof of the fact that (d) implies (c).

$(\gamma)$ To show that (b) implies (d), we may replace $B_{X}$. by a weak*-compact convex subset $K$ of $X^{*}$ in Janicka's proof in [9] (see the remark preceding Theorem I in §1). Finally, it is quite clear that (b) implies (a). Hence the proof is completed.

The proof of Proposition 1 combined with Theorems I, II and III yields the following Proposition 2. In the statement (b) of Proposition 2, $[0,1]$ is endowed with $\Lambda$ and $\lambda$.

Proposition 2. Let $K$ be a weak*-compact convex subset of $X^{*}$. Then the following statements about $K$ are equivalent.

(a) The set $K$ has the WRNP.

(b) For any weak*-measurable function $f:[0,1] \rightarrow K, f$ is weak* equivalent to a weakly measurable function $g:[0,1] \rightarrow K$.

(c) For any $(\Omega, \Sigma, \mu)$ and any weak*-measurable function $f: \Omega \rightarrow K, f$ is weak* equivalent to a weakly measurable function $g: \Omega \rightarrow K$.

Proof. ( $\alpha$ ) First let us prove that (a) implies (c). Let $f: \Omega \rightarrow K$ be a weak*- 
measurable function. Consider a measure $\kappa: \Sigma \rightarrow X^{*}$ defined for each $E \in \Sigma$ by putting

$$
(\kappa(E), x)=\int_{E}(f(\omega), x) d \mu(\omega)
$$

for every $x \in X$. Then, by the separation theorem, $\kappa(E) \in \mu(E) \cdot K$ for each $E \in \Sigma$. Hence there exists a Pettis integrable (so weakly measurable) function $g: \Omega \rightarrow K$ such that

$$
\left(x^{* *}, \kappa(E)\right)=\int_{E}(x * *, g(\omega)) d \mu(\omega)
$$

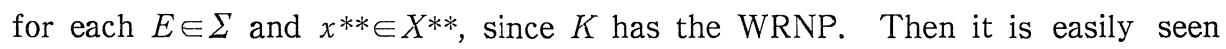
that $f$ is weak* equivalent to $g$, and so $g$ is a desired weakly measurable function.

( $\beta$ ) Next let us prove that (b) implies (a). This part is principal in our proof of Proposition 2. Suppose that (a) fails. Then, from the first part of the proof of Proposition 1, we have a compact metrizable space $T *(K)$ and an independent sequence of pairs $\left(C_{n}, D_{n}\right)_{n \geq 1}$, where $C_{n}$ and $D_{n}$ are closed subsets of $T *(K)$. Hence, by virtue of Theorem II, we can choose $\Gamma \subset T^{*}(K), n(1)<n(2)$ $<\cdots$ and a homeomorphism $\sigma$ from $\Gamma$ onto $\Delta$ such that $C_{n(k)} \cap \Gamma=\sigma^{-1}\left(U_{k}\right)$ and $D_{n(k)} \cap \Gamma=\sigma^{-1}\left(U_{k}^{c}\right)$ for all $k$. Set $\phi=\sigma^{-1}: \Delta \rightarrow \Gamma$. Then for every weak*-cluster point $L_{\mathscr{F}}$ of a sequence $\left\{e_{n(k)}\right\}_{k \geqq 1}$ in $l_{\infty}^{*}, L_{\mathscr{T}}$ is not measurable for $\mu(=\psi(\nu))$ on $\Gamma$. Here $\nu$ denotes the normalized Haar measure on $\Delta$. Moreover we have a measure $\xi: \Lambda \rightarrow l_{\infty}$ defined for each $E \in \Lambda$ by putting

$$
(\xi(E), a)=\int_{E}(\psi(\phi(t)), a) d \lambda(t)
$$

for every $a \in l_{1}$, where $\phi:[0,1] \rightarrow \Delta$ denotes the Sierpinski's function (see $\S 1$ in [10]). Then we have $\xi(E) \in \lambda(E) \cdot T *(K)$ for each $E \in \Lambda$. So, in virtue of Theorem III, there exists a measure $\beta: \Lambda \rightarrow X^{*}$ such that $\beta(E) \in \lambda(E) \cdot K$ and $T^{*} \beta(E)=\xi(E)$ for each $E \in \Lambda$. Hence, by Theorem I, there exists a weak*measurable function $f:[0,1] \rightarrow K$ such that

$$
(\beta(E), x)=\int_{E}(f(t), x) d \lambda(t)
$$

for each $E \in \Lambda$ and $x \in X$. Suppose that there exists a weakly measurable function $g:[0,1] \rightarrow K$ which is weak* equivalent to $f$. Then we have

$$
\begin{aligned}
(\xi(E), a) & =\left(T^{*} \beta(E), a\right)=(\beta(E), T a)=\int_{E}(f(t), T a) d \lambda(t) \\
& =\int_{E}(g(t), T a) d \lambda(t)=\int_{E}\left(T^{*} g(t), a\right) d \lambda(t)
\end{aligned}
$$

for each $E \in \Lambda$ and $a \in l_{1}$, since $g$ is weak* equivalent to $f$. Hence we have

$$
\int_{E}(\psi(\phi(t)), a) d \lambda(t)=\int_{E}\left(T^{*} g(t), a\right) d \lambda(t)
$$


for each $E \in \Lambda$ and $a \in l_{1}$. This yields that $\phi(\phi(t))=T^{*} g(t) \lambda$-a. e., since $l_{1}$ is separable. Thus $\left(L_{\mathscr{T}}, \phi(\phi(t))\right)=\left(L_{\mathscr{F}}, T^{*} g(t)\right)=\left(T^{* *} L_{\mathscr{F}}, g(t)\right) \lambda$-a. e. and so $\left(L_{\mathscr{q}}\right.$, $\psi(\phi(t)))$ is measurable for $\lambda$, since $g$ is weakly measurable. Consequently $L_{q}$ is measurable for $\phi(\phi(\lambda))=\mu$, which is a contradiction. Hence we complete the proof of the fact that (b) implies (a). Moreover, the fact that (c) implies (b) is obvious and so the proof is completed.

Remark 1. Without invoking Theorem II, the equivalence between the statements (a) and (c) of Proposition 2 can be proved. In fact, we have only to show that (c) implies (a), whose proof goes as follows. Suppose that (a) fails. Then, by the second part of the proof of Proposition 1, we have a complete finite measure space $\left(T *(K), \mathfrak{M}_{\gamma}, \gamma\right)$ and a measure $\beta: \mathfrak{M}_{\gamma} \rightarrow X^{*}$ such that $\beta(E) \in$ $\gamma(E) \cdot K$ and $T^{*} \beta(E)=\alpha(E)$ for each $E \in \mathfrak{M}_{\gamma}$. Hence, by Theorem I, there exists a weak*-measurable function $f: T^{*}(K) \rightarrow K$ such that

$$
(\beta(E), x)=\int_{E}\left(f\left(a^{*}\right), x\right) d \gamma\left(a^{*}\right)
$$

for each $E \in \mathfrak{M}_{\gamma}$ and $x \in X$. Then, by the same argument as in the proof of Proposition 2, we know that $f$ is not weak* equivalent to any weakly measurable function $g: T *(K) \rightarrow K$. So (c) fails.

\section{§3. Weak Pre-Compactness, Complete Continuity and the Weak Radon-Nikodym Property}

A subset $H$ of $X$ is called weakly pre-compact if every bounded sequence in $H$ has a weakly Cauchy subsequence, that is, every bounded sequence $\left\{x_{n}\right\}_{n \geqq 1}$ in $H$ has a subsequence $\left\{x_{n(k)}\right\}_{k \geqq 1}$ such that for every $x^{*} \in X^{*}, \lim _{k \rightarrow \infty}\left(x^{*}, x_{n(k)}\right)$ exists. As stated in Rosenthal's fundamental paper [18], the notion of weak pre-compactness is very important on the study of Banach spaces not containing a copy of $l_{1}$ (as well as dual Banach spaces with the WRNP). Therefore it is natural for us to find out a corresponding notion which is convenient for the study of weak*-compact convex sets with the WRNP. Suggested by the statement (f) in Theorem D, we introduce a following notion of relative weakly precompact sets.

Definition 2. A subset $H$ of $X$ is called weakly pre-compact with respect to a subset $K$ of $X^{*}$ if every bounded sequence $\left\{x_{n}\right\}_{n \geqq 1}$ in $H$ has a subsequence $\left\{x_{n(k)}\right\}_{k \geqq 1}$ such that for every $x^{*} \in K, \lim _{k \rightarrow \infty}\left(x^{*}, x_{n(k)}\right)$ exists.

Expressing the weak pre-compactness of $H$ in our notion, $H$ is weakly precompact with respect to $B_{X^{*}}$, and the statement $(\mathrm{h})$ in Theorem $\mathrm{E}$ can be expressed in the way that $B_{X}$ is weakly pre-compact with respect to $K$.

A subset $H$ of $X$ is called a set of complete continuity with respect to 
$(\Omega, \Sigma, \mu)$ if for every bounded linear operator $S: L_{1}(\Omega, \Sigma, \mu) \rightarrow X$ for which $S\left(\chi_{E} / \mu(E)\right)$ belongs to $H$ for each non null set $E \in \Sigma$, the operator $S$ is a DunfordPettis operator. We say that a set $H$ is a set of complete continuity if $H$ has this property with respect to every $(\Omega, \Sigma, \mu)$. There is a well-known observation on Dunford-Pettis operators in terms of vector measures (cf. [16], p. 530), which is used in the following.

Observation. Let $S: L_{1}(\Omega, \Sigma, \mu) \rightarrow X$ be a bounded linear operator. Then the operator $S$ is a Dunford-Pettis operator if and only if the vector measure $\alpha$ defined for each $E \in \Sigma$ by $\alpha(E)=S\left(\chi_{E}\right)$ has a relatively norm compact range.

First, concerning the relative weak pre-compactness defined above, we have:

Proposition 3. A bounded subset $H$ of $X$ is weakly pre-compact with respect to a weak*-compact convex subset $K$ of $X^{*}$ if and only if for every $(\Omega, \Sigma, \mu)$ and every bounded linear operator $S: X \rightarrow L_{\infty}(\Omega, \Sigma, \mu)$ such that $S *\left(\chi_{E}\right) \in \mu(E) \cdot K$ for each $E \in \Sigma, S$ takes sequences in $H$ into sequences with almost everywhere convergent subsequences.

This Proposition 3 can be regarded as a localization and extension of Theorem 1 in [16]. Next we want to show the equivalence among the statements (c), (d), (e), (f) and (h) in Theorem E. That is, we have:

Proposition 4. Let $K$ be a weak*-compact convex subset of $X^{*}$. Then cach of the following statements about $K$ implies all the others.

(a) The set $K$ is a set of complete continuity.

(b) The set $K$ is a set of complete continuity with respect to $([0,1], \Lambda, \lambda)$.

(c) The set $K$ has the WRNP.

(d) The set $K$ has the WRNP with respect to ([0,1], $\Lambda, \lambda$ ).

(e) The set $B_{X}$ is weakly pre-compact with respect to $K$.

Before proving Propositions 3 and 4, we first prepare some lemmas. Viewing from our standpoint, Lemmas 3, 4 and ${ }_{5}^{8} 5$ below are fundamental. Before stating them, we need the

Definition 3 ([18]). Let $\left(A_{n}, B_{n}\right)_{n \geqq 1}$ be a sequence of pairs of subsets of some set $S$ with $A_{n} \cap B_{n}=\phi$ for all $n$. We say that $\left(A_{n}, B_{n}\right)_{n \geqq 1}$ converges if every point $s \in S$ belongs at most finitely many $A_{n}$ 's or finitely many $B_{n}$ 's.

Lemma 2 (Lemma 2.4 in [19]). Let $\left\{f_{n}\right\}_{n \geq 1}$ be a point-wise bounded sequence of real-valued functions defined on a set $S$ and having no point-wise convergent subsequence. Then there exist a subsequence $\left\{f_{n(k)}\right\}_{k \geqq 1}$ of $\left\{f_{n}\right\}_{n \geqq 1}$ and real numbers $r$ and $\delta$ with $\delta>0$ such that for every infinite subset $M$ of $\{n(k): k \geqq 1\}$, there is an $s \in S$ with 
and

$$
f_{m}(s)>r+\delta \text { for infinitely many } m \in M
$$

$$
f_{m}(s)<r \quad \text { for infinitely many } m \in M \text {. }
$$

Lemma 3. Let $X$ be a separable Banach space and $K$ a weak*-compact convex subset of $X^{*}$. Suppose that there exists a sequence $\left\{x_{n}\right\}_{n \geqq 1}$ in $B_{X}$ having no point-wise convergent subsequence on $K$ (namely, $B_{X}$ is not weakly pre-compact with respect to $K)$. Then there exist a complete finite measure space $(\Omega, \Sigma, \mu)$ and $a$ bounded linear operator $U: L_{1}(\Omega, \Sigma, \mu) \rightarrow X^{*}$ such that

(1) $U\left(\chi_{E}\right) \in \mu(E) \cdot K$ for each $E \in \Sigma$,

(2) $\left\{U\left(\chi_{E}\right): E \in \Sigma\right\}$ is not relatively norm compact.

Proof of Lemma 3 using Theorem II and Lemma 2. By virtue of Lemma 2, there exist a subsequence $\left\{x_{n(k)}\right\}_{k \geqq 1}$ of $\left\{x_{n}\right\}_{n \geqq 1}$ and real numbers $r$ and $\delta$ with $\delta>0$ such that for every infinite subset $M$ of $\{n(k): k \geqq 1\}$, there is an $x^{*} \in K$ with

and

$$
\left(x^{*}, x_{m}\right)>r+\delta \text { for infinitely many } m \subseteq M
$$

$$
\left(x^{*}, x_{m}\right)<r \quad \text { for infinitely many } m \in M \text {. }
$$

Putting $A_{k}=\left\{x^{*} \in K:\left(x^{*}, x_{n(k)}\right) \geqq r+\delta\right\}$ and $B_{k}=\left\{x * \in K:\left(x^{*}, x_{n(k)}\right) \leqq r\right\},\left(\mathcal{A}_{k}\right.$, $\left.B_{k}\right)_{k \geqq 1}$ is a sequence of pairs of subsets of $K$ with $A_{k}, B_{k}$ closed and $A_{k} \cap B_{k}=\phi$ for all $k$. Further $\left(A_{k}, B_{k}\right)_{k \geqq 1}$ has no convergent subsequence. As $K$ is a compact metrizable space, by virtue of Theorem II, there exist a compact subset $\Gamma$ of $K$ homeomorphic to $\Delta$, a homeomorphism $\sigma$ from $\Gamma$ onto $\Delta$, and a sequence $k(1)<k(2)<\cdots$ such that $A_{k(\imath)} \cap \Gamma=\sigma^{-1}\left(U_{\imath}\right)$ and $B_{k(\imath)} \cap \Gamma=\sigma^{-1}\left(U_{\imath}^{c}\right)$ for all $i$. Let $\nu$ be the normalized Haar measure on $\Delta$. Then there exists a Radon probability measure $\rho$ on $\Gamma$ such that $\sigma(\rho)=\nu$. Denote by $\bar{\rho}$ the Radon probability measure on $K$ extending $\rho$. Then $\bar{\rho}\left(A_{k(\imath)}\right)=\rho\left(A_{k(\imath)} \cap \Gamma\right)=\rho\left(\sigma^{-1}\left(U_{\imath}\right)\right)=\sigma(\rho)\left(U_{\imath}\right)=\nu\left(U_{\imath}\right)=1 / 2$ $=\bar{\rho}\left(B_{k(\imath)}\right)$ and $\bar{\rho}\left(A_{k(\imath)} \cap B_{k(\jmath)}\right)=\rho\left(A_{k(\imath)} \cap B_{k(\jmath)} \cap \Gamma\right)=\rho\left(\sigma^{-1}\left(U_{\imath} \cap U_{\jmath}^{c}\right)\right)=\sigma(\rho)\left(U_{\imath} \cap U_{\imath}^{c}\right)$ $=\nu\left(U_{\imath} \cap U_{\jmath}^{c}\right)=1 / 4=\bar{\rho}\left(A_{k(\jmath)} \cap B_{k(\imath)}\right)$ for $i<j$.

Consider the complete finite measure space $\left(K, \mathfrak{M}_{\bar{\rho}}, \bar{\rho}\right)$ and a meas'ure $\alpha^{\prime}: \mathfrak{M}_{\bar{\rho}}$ $\rightarrow X^{*}$ defined for each $E \in \mathfrak{M}_{\bar{\rho}}$ by putting

$$
(\alpha(E), x)=\int_{E}\left(x^{*}, x\right) d \bar{\rho}\left(x^{*}\right)
$$

for every $x \in X$. Then, by the separation theorem, $a(E) \in \bar{\rho}(E) \cdot K$ for each $E \in \mathfrak{M}_{\bar{\rho}}$. Further we have for $i<j$

$$
\begin{aligned}
& \left(\alpha\left(A_{k(\imath)}\right)-\alpha\left(A_{k(\jmath)}\right), x_{n(k(\imath))}\right) \\
& =\int_{A_{k(2)}}\left(x^{*}, x_{n(k(2))^{\prime}} d \bar{\rho}\left(x^{*},-\int_{A_{k(3)}}\left(x^{*}, \tilde{x}_{n(k(2))}\right) d \bar{\rho}^{\prime}\left(x^{*},\right.\right.\right.
\end{aligned}
$$

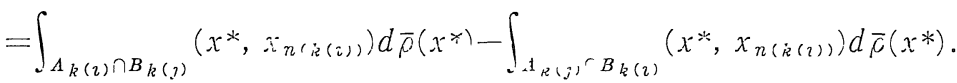


Let $a^{*} \in A_{k(i)} \cap B_{k(\jmath)}$ and $b^{*} \in A_{k(j)} \cap B_{k(i)}$. Then we have $\left(a^{*}, x_{n(k(i))}\right) \geqq r+\delta$ and $-\left(b^{*}, x_{n(k(i))}\right) \geqq-r$. Therefore we have

$$
\begin{aligned}
\left\|\alpha\left(A_{k(i)}\right)-\alpha\left(A_{k(\jmath)}\right)\right\| & \geqq\left(\alpha\left(A_{k(i)}\right)-\alpha\left(A_{k(j)}\right), x_{n(k(i))}\right) \\
& \geqq(r+\delta) \cdot \bar{\rho}\left(A_{k(i)} \cap B_{k(\jmath)}\right)-r \cdot \bar{\rho}\left(A_{k(\jmath)} \cap B_{k(i)}\right) \\
& =\delta / 4 \quad \text { for } i<j .
\end{aligned}
$$

Thus, by the standard argument, the vector measure $\alpha$ induces a bounded linear operator $U: L_{1}\left(K, \mathfrak{M}_{\bar{\rho}}, \bar{\rho}\right) \rightarrow X^{*}$ that satisfies

(1) $U\left(\chi_{E}\right) \in \bar{\rho}(E) \cdot K$ for each $E \in \mathfrak{M}_{\bar{\rho}}$,

(2) $\left\{U\left(\chi_{E}\right): E \in \mathfrak{M}_{\bar{\rho}}\right\}$ is not relatively norm compact.

So the proof is completed.

Suggested by this Lemma 3, we become aware of the following Lemma 4 . This can be shown by modifying the proof of Lemma 3 .

Lemma 4. Assume the same conditions as in Lemma 3. Then there is a bounded linear operator $V: L_{1}([0,1], \Lambda, \lambda) \rightarrow X^{*}$ such that

(1) $V\left(\chi_{E}\right) \in \lambda(E) \cdot K$ for each $E \in \Lambda$,

(2) $\left\{V\left(\chi_{E}\right): E \in \Lambda\right\}$ is not relatively norm compact.

Proof. Under the same notations as in Lemma 3, let $\phi=\sigma^{-1}: \Delta \rightarrow \Gamma$. Then $\phi\left(U_{i}\right)=A_{k(i)} \cap \Gamma$ and $\phi\left(U_{i}^{c}\right)=B_{k(i)} \cap \Gamma$. Let $\phi:[0,1] \rightarrow \Delta$ be the Sierpinski's function. Consider a measure $\xi: \Lambda \rightarrow X^{*}$ defined for each $E \in \Lambda$ by putting

$$
(\xi(E), x)=\int_{E}(\phi(\phi(t)), x) d \lambda(t)
$$

for every $x \in X$. Then we have $\xi(E) \in \lambda(E) \cdot K$ for each $E \in \Lambda$. Furthermore we have

$$
\begin{aligned}
\left(\xi\left(\phi^{-1}(B)\right), x\right) & =\int_{\phi^{-1}(B)}(\phi(\phi(t)), x) d \lambda(t) \\
& =\int_{B}(\phi(s), x) d \phi(\lambda)(s)=\int_{B}(\psi(s), x) d \nu(s)
\end{aligned}
$$

for each $B \in \mathfrak{B}(\mathcal{d})$, making use of the change-of-variables formula. Hence we have for $i<j$

$$
\begin{aligned}
& \left(\xi\left(\dot{\phi}^{-1}\left(U_{i}\right)\right), x_{n(k(\imath))}\right)-\left(\xi\left(\phi^{-1}\left(U_{\jmath}\right)\right), x_{n(k(\imath))}\right) \\
& =\int_{U_{i}}\left(\psi(s), x_{n(k(\imath))}\right) d \nu(s)-\int_{U_{j}}\left(\psi(s), x_{n(k(\imath))}\right) d \nu(s) \\
& =\int_{U_{\imath} \backslash U_{j}}\left(\psi(s), x_{n(k(\imath))}\right) d \nu(s)-\int_{U_{j}\left(U_{i}\right.}\left(\psi(s), x_{n(k(\imath))}\right) d \nu(s) .
\end{aligned}
$$

Let $s_{1} \in U_{\imath} \backslash U_{\jmath}$ and $s_{2} \in U_{\jmath} \backslash U_{i}$. Then we have $\left(\phi\left(s_{1}\right), x_{n(k(i))}\right) \geqq r+\delta$ and $-\left(\phi\left(s_{2}\right)\right.$, $\left.x_{n(k(i))}\right) \geqq-r$. Thus we have 


$$
\begin{aligned}
\left\|\xi\left(\phi^{-1}\left(U_{\imath}\right)\right)-\xi\left(\phi^{-1}\left(U_{\jmath}\right)\right)\right\| & \geqq\left(\xi\left(\phi^{-1}\left(U_{\imath}\right)\right), x_{n(k(\imath))}\right)-\left(\xi\left(\phi^{-1}\left(U_{\jmath}\right)\right), x_{n(k(i))}\right) \\
& \geqq(r+\delta) / 4-r / 4=\delta / 4 \quad \text { for } i<j,
\end{aligned}
$$

Consequently, the vector measure $\xi$ induces a bounded linear operator $V: L_{1}([0$, 1], $\Lambda, \lambda) \rightarrow X^{*}$ such that

(1) $V\left(\chi_{E}\right) \in \lambda(E) \cdot K$ for each $E \in \Lambda$,

(2) $\left\{V\left(\chi_{E}\right): E \in \Lambda\right\}$ is not relatively norm compact.

Thus the proof is completed.

Lemma 5. Assume the same conditions as in Lemma 3. Then there exist a complete finite measure space $(\Omega, \Sigma, \mu)$ and a bounded linear operator $S: X \rightarrow$ $L_{\infty}(\Omega, \Sigma, \mu)$ such that

(1) $S *\left(\chi_{E}\right) \in \mu(E) \cdot K$ for each $E \in \Sigma$,

(2) There exists a subsequence $\left\{x_{m(\jmath)}\right\}_{\jmath \geqq 1}$ of $\left\{x_{n}\right\}_{n \geqq 1}$ such that $\left\{S x_{m(\jmath)}\right\}_{\jmath \geqq 1}$ has no almost everywhere convergent subsequence.

Proof. The proof of Lemma 3 shows that there exist a complete finite measure space $\left(K, \mathfrak{M}_{\bar{\rho}}, \bar{\rho}\right)$ and a bounded linear operator $U: L_{1}\left(K, \mathfrak{M}_{\bar{\rho}}, \bar{\rho}\right) \rightarrow X^{*}$ satisfying $U\left(\chi_{E}\right) \in \bar{\rho}(E) \cdot K$ for each $E \in \mathfrak{M}_{\bar{\rho}}$. Define a bounded linear operator $S: X \rightarrow L_{\infty}\left(K, \mathfrak{M}_{\bar{\rho}}, \bar{\rho}\right)$ by $S x=x_{\mid K}$ for every $x \in X$. Then we have

$$
\left(S *\left(\chi_{E}\right), x\right)=\left(\chi_{E}, S x\right)=\int_{E}\left(x^{*}, x\right) d \bar{\rho}(x *)=\left(U\left(\chi_{E}\right), x\right)
$$

for each $E \in \mathfrak{M}_{\bar{\rho}}$ and $x \in X$. So $S *\left(\chi_{E}\right)=U\left(\chi_{E}\right)$ for each $E \in \mathfrak{M}_{\bar{\rho}}$. Moreover, for a subsequence $\left\{x_{n(k(i))}\right\}_{i \geqq 1}$ of $\left\{x_{n}\right\}_{n \geq 1}$ in Lemma $3, S x_{n(k(i))}=x_{n(k(i)) \mid K}$ has no almost everywhere convergent subsequence. So the proof is completed.

For convenient reference we remark here that Theorem III easily yields the following lifting theorem for operators: Let $T: X \rightarrow Y$ be a bounded linear operator. If $K$ is a weak*-compact convex subset of $Y^{*}$, then for every bounded linear operator $U: L_{1}(\Omega, \Sigma, \mu) \rightarrow X^{*}$ such that $U\left(\chi_{E}\right) \in \mu(E) \cdot T *(K)$ for each $E \in \Sigma$, there exists a bounded linear operator $V: L_{1}(\Omega, \Sigma, \mu) \rightarrow Y^{*}$ such that $V\left(\chi_{E}\right) \in$ $\mu(E) \cdot K$ for each $E \in \Sigma$ and $T * V(f)=U(f)$ for each $f \in L_{1}(\Omega, \Sigma, \mu)$.

Now let us prove Propositions 3 and 4 .

Proof of Proposition 3. (Sufficiency). This is the main part of this proof. Without loss of generality, we may assume that $H \subset B_{X}$. Suppose the contrary. Then there exists a sequence $\left\{x_{n}\right\}_{n \geq 1}$ in $H$ which has no point-wise convergent subsequence on $K$. Let $X_{0}$ denote the closed linear span of the set $\left\{x_{n}: n \geqq 1\right\}$ and let $j: X_{0} \rightarrow X$ be the inclusion map. Then a sequence $\left\{x_{n}\right\}_{n \geq 1}$ in $B_{X_{0}}$ has no point-wise convergent subsequence on $j *(K)$. Hence, by virtue of Lemma 5, there exist a complete finite measure space $(\Omega, \Sigma, \mu)$ and a bounded linear operator $T: X_{0} \rightarrow L_{\infty}(\Omega, \Sigma, \mu)$ such that

(1) $T *\left(\chi_{E}\right) \in \mu(E) \cdot j *(K)$ for each $E \in \Sigma$, 
(2) There exists a subsequence $\left\{x_{n(k)}\right\}_{k \geqq 1}$ of $\left\{x_{n}\right\}_{n \geqq 1}$ such that $\left\{T x_{n(k)}\right\}_{k \geqq 1}$ has no almost everywhere convergent subsequence.

Then, by the preceding remark, there exists a bounded linear operator $U$ : $L_{1}(\Omega, \Sigma, \mu) \rightarrow X^{*}$ such that

(3) $U\left(\chi_{E}\right) \in \mu(E) \cdot K$ for each $E \in \Sigma$,

(4) $j * U(f)=T *(f)$ for each $f \in L_{1}(\Omega, \Sigma, \mu)$.

Define a bounded linear operator $S: X \rightarrow L_{\infty}(\Omega, \Sigma, \mu)$ by $S x=x \circ h$ for every $x \in X$, where $h: \Omega \rightarrow K$ is a weak*-measurable function satisfying

$$
\left(U\left(\chi_{E}\right), x\right)=\int_{E}(h(\omega), x) d \mu(\omega)
$$

for each $E \in \Sigma$ and $x \in X$. The existence of such an $h$ is assured by Theorem I. Then we have for every $k$

$$
\left(j * U\left(\chi_{E}\right), x_{n(k)}\right)=\left(T *\left(\chi_{E}\right), x_{n(k)}\right)=\left(\chi_{E}, T x_{n(\xi)}\right)=\int_{E} T x_{n(k}(\omega) d \mu(\omega) .
$$

On the other hand, we have for every $k$

$$
\left(j * U\left(\chi_{E}\right), x_{n(k)}\right)=\left(U\left(\chi_{E}\right), j x_{n(k)}\right)=\int_{E}\left(h(\omega), x_{n(k)}\right) d \mu(\omega)=\int_{E} S x_{n(k)}(\omega) d \mu(\omega) .
$$

Thus we have $S x_{n(k)}=T x_{n(k)}$ in $L_{\infty}(\Omega, \Sigma, \mu)$. Moreover we have

$$
\left(S *\left(\chi_{E}\right), x\right)=\left(\chi_{E}, S x\right)=\int_{E}(h(\omega), x) d \mu(\omega)=\left(U\left(\chi_{E}\right), x\right)
$$

for each $x \in X$. So $S *\left(\chi_{E}\right)=U\left(\chi_{E}\right) \in \mu(E) \cdot K$ for each $E \in \Sigma$. The existence of such an operator $S$ contradicts our assumption. Hence the proof of this part is completed.

(Necessity). In view of Theorem I, the proof of this part can be given by the same argument as in the necessity part of Theorem 1 in [16]. That is, we may replace $B_{X}$. by a weak*-compact convex subset $K$ of $X^{*}$ in that place. Hence we omit it.

Remark 2. Adopting the same argument as in Lemmas 4 and 5, we can deduce the following: Assume the same conditions as in Lemma 3. Then there exists a bounded linear operator $S: X \rightarrow L_{\infty}([0,1], \Lambda, \lambda)$ such that

(1) $S *\left(\chi_{E}\right) \in \lambda(E) \cdot K$ for each $E \in \Lambda$,

(2) There exists a subsequence $\left\{x_{n(\jmath)}\right\}_{\jmath \unrhd 1}$ of $\left\{x_{n}\right\}_{n \geq 1}$ such that $\left\{S x_{n(\jmath)}\right\}_{\jmath \geqq 1}$ has no almost everywhere convergent subsequence.

Hence, by the same argument as in Proposition 3, we have that a bounded subset $H$ of $X$ is weakly pre-compact with respect to a weak*-compact convex subset $K$ of $X^{*}$ if and only if for every bounded linear operator $S: X \rightarrow L_{\infty}([0,1], \Lambda, \lambda)$ such that $S^{*}\left(\chi_{E}\right) \in \lambda(E) \cdot K$ for each $E \in \Lambda, S$ takes sequences in $H$ into sequences with almost everywhere convergent subsequence. This is a slight refinement of Proposition 3. 
Proof of Proposition 4. ( $\alpha$ ) First let us prove that (b) implies (e). This is the crucial part of our proof. Suppose that (e) fails. Then there exists a sequence $\left\{x_{n}\right\}_{n \geq 1}$ in $B_{X}$ such that $\left\{x_{n}\right\}_{n \geqq 1}$ has no point-wise convergent subsequence on $K$. Let $X_{0}$ denote the closed linear span of the set $\left\{x_{n}: n \geqq 1\right\}$, and let $j: X_{0} \rightarrow X$ be the inclusion map. Then a sequence $\left\{x_{n}\right\}_{n \geqq 1}$ in $B_{X_{0}}$ has no point-wise convergent subsequence on $j *(K)$. Hence, in virtue of Lemma 4, there exists a bounded linear operator $V: L_{1}([0,1], \Lambda, \lambda) \rightarrow X_{0}^{*}$ such that

(1) $V\left(\chi_{E}\right) \in \lambda(E) \cdot j *(K)$ for each $E \in \Lambda$,

(2) $\left\{V\left(\chi_{E}\right): E \in \Lambda\right\}$ is not relatively norm compact.

By the remark preceding Proof of Proposition 3, there exists a bouncied linear operator $S: L_{1}([0,1], \Lambda, \lambda) \rightarrow X^{*}$ such that

(3) $S\left(\chi_{E}\right) \in \lambda(E) \cdot K$ for each $E \in \Lambda$,

(4) $j * S(f)=V(f)$ for each $f \in L_{1}([0,1], \Lambda, \lambda)$.

The statement (4) combined with the statement (2) proves that $\left\{j * S\left(\chi_{E}\right): E \in \Lambda\right\}$ is not relatively norm compact and so is $\left\{S\left(\chi_{E}\right): E \in \Lambda\right\}$. Hence the operator $S$ is not a Dunford-Pettis operator by the above Observation. Thus (b) fails.

( $\beta$ ) Next let us prove that (e) implies (a). In view of Theorem I, the proof of this part can be given by the same argument as in the first part of the proof of Pelczynski's Theorem in [17]. That is, we may replace $B_{X^{*}}$ by a weak*compact convex subset $K$ of $X^{*}$ in that place. Hence we omit this proof.

$(\gamma)$ Thus we see that the statements (a), (b) and (e) are equivalent since it is clear that (a) implies (b). To prove that (d) implies (e), suppose that (e) fails. Then, by $(\alpha)$, there exists a measure $\xi: \Lambda \rightarrow X^{*}$ such that $\xi(E) \in \lambda(E) \cdot K$ for each $E \in \Lambda$ and $\{\xi(E): E \in \Lambda\}$ is not relatively norm compact. Since $K$ has the WRNP with respect to $([0,1], \Lambda, \lambda), \xi$ can be represented as an indefinite Pettis integral. Since $([0,1], \Lambda, \lambda)$ is a perfect measure space, Stegall's theorem (Proposition $3 \mathrm{~J}$ in [6]) ensures that $\xi$ has a relatively norm compact range, which is a contradiction.

$(\boldsymbol{\delta})$ Since it is clear that (c) implies (d), we have only to show that (e) implies (c) to complete the proof of Proposition 4. Suppose that (c) fails. By Proposition 1, there exists an element $x^{* *}$ of $X^{* *}$ that does not satisfy the barycentric calculus on $K$. Then, by virtue of Lemma 1 , there exist a nonempty compact subset $S$ of $K$, real numbers $r$ and $\delta$ with $\delta>0$ and a bounded sequence $\left\{x_{n}\right\}_{n \geqq 1}$ in $X$ such that $\left(A_{n}, B_{n}\right)_{n \geqq 1}$ is independent, where $A_{n}=\left\{x^{*} \in\right.$ $\left.S:\left(x^{*}, x_{n}\right) \geqq r+\delta\right\}$ and $B_{n}=\left\{x^{*} \in S:\left(x^{*}, x_{n}\right) \leqq r\right\}$. So $\bigcap_{n=1}^{\infty} \varepsilon_{n} A_{n} \neq \phi$ for all $\left\{\varepsilon_{n}\right\}_{n \geqq 1}$ with $\varepsilon_{n}= \pm 1$. This means that $\left\{x_{n}\right\}_{n \geqq 1}$ has no point-wise convergent sulsequence on $K$. Hence (e) fails.

The following Corollaries 1 and 2 easily follow from Propcsition 4. Since it easily follows from the definition of relative weak pre-compactness that given a bounded linear operator $T: X \rightarrow Y, T\left(B_{X}\right)$ is weakly pre-compact with respect to $K$ if and only if $B_{X}$ is weakly pre-compact with respect to $T^{*}\left(K^{\prime}\right)$, we frst 
have :

Corollary 1. Let $T: X \rightarrow Y$ be a bounded linear operator and let $K$ be $a$ weak*-compact convex subset of $Y^{*}$. Then each of the following statements about $T$ and $K$ implies all the others.

(a) The set $T\left(B_{X}\right)$ is weakly pre-compact with respect to $K$.

(b) The set $T^{*}(K)$ is a set of complete continuity.

(c) The set $T *(K)$ is a set of complete continuity with respect to $([0,1], \Lambda, \lambda)$.

(d) The set $T *(K)$ has the WRNP.

(e) The set $T^{*}(K)$ has the WRNP with respect to $([0,1], \Lambda, \lambda)$.

Setting $K=B_{Y^{*}}$ in Corollary 1 , we have :

Corollary 2 (cf. Theorem C). Let $T: X \rightarrow Y$ be a bounded linear operator. Then each of the following statements about $T$ implies all the others.

(a) The set $T\left(B_{X}\right)$ is weakly pre-compact.

(b) The set $T^{*}\left(B_{Y^{*}}\right)$ is a set of complete continuity.

(c) The set $T^{*}\left(B_{Y^{*}}\right)$ is a set of complete continuity with respect to $([0,1], \Lambda, \lambda)$.

(d) The set $T^{*}\left(B_{Y^{*}}\right)$ has the WRNP.

(e) The set $T^{*}\left(B_{Y^{*}}\right)$ has the WRNP with respect to $([0,1], \Lambda, \lambda)$.

Applying the equivalence between the statements (c) and (e) in Proposition 4, we have:

Corollary 3. Let $K$ be a weak*-compact convex subset of $X^{*}$. Then the set $K$ has the WRNP if and only if for every closed separable subspace $Y$ of $X$, the set $j *(K)$ has the WRNP (where $j$ is the inclusion map: $Y \rightarrow X$ ).

Proof. Only if part easily follows from Theorem III (or, the equivalence between the statements (a) and (d) in Proposition 1, see Corollary 2 in [22]). If part can be proved in the following way. Suppose the contrary. Then, by Proposition 4 , there exists a sequence $\left\{x_{n}\right\}_{n \geq 1}$ in $B_{X}$ having no point-wise convergent subsequence on $K$. Let $Y$ be the closed linear span of $\left\{x_{n}: n \geqq 1\right\}$. Then $Y$ is a closed separable subspace of $X$ and a sequence $\left\{x_{n}\right\}_{n \geqq 1}$ in $B_{Y}$ has no point-wise convergent subsequence on $j *(K)$, which implies that $j *(K)$ does not have the WRNP by Proposition 4. So the proof is completed.

This Corollary 3 is convenient for the study of the relation between the scalar point of continuity property and the WRNP in $\S 4$.

\section{§4. Scalar Point of Continuity Property and the Weak Radon-Nikodym Property}

Let $K$ be a weak*-compact convex subset of $X^{*}$. Following Riddle, Saab 
and Uhr [16], we say that $K$ has the scalar point of continuity property if for each weak*-compact subset $M$ of $K$ and every $x^{* *} \in X^{* *}$, the restriction of $x^{* *}$ to $M$ has a point of continuity. Here $M$ is equipped with the weak*-topology. We first remark concerning this notion that if $K$ does not have the scalar point of continuity property, then there exist a non-empty compact subset $S$ of $K$, real numbers $r$ and $\delta$ with $\delta>0$ and a bounded sequence $\left\{x_{n}\right\}_{n \geqq 1}$ in $X$ such that putting $A_{n}=\left\{x^{*} \in S:\left(x^{*}, x_{n}\right) \geqq r+\delta\right\}$ and $B_{n}=\left\{x^{*} \in S:\left(x^{*}, x_{n}\right) \leqq r\right\}$, then $\left(A_{n}\right.$, $\left.B_{n}\right)_{n \geqq 1}$ is independent, which easily follows from Lemmas 2 and 3 in [13] (that is, this statement is a special case of Lemmas 2 and 3 in [13]). This fact indicates the relation between the scalar point of continuity property and the weak pre-compactness. Based on this observation, we show in this section that the equivalence between the statements (e) and ( $\mathrm{g}$ ) in Theorem $\mathrm{E}$ easily follows from the separable case, making use of our Corollary 3. In fact, in the case where $X$ is separable, we notice that the Baire characterization theorem on the first Baire class (see [4], Chapter 7, Theorem 2, p. 67 or [13], p. 376) is very available for the study of the relation between the scalar point of continuity property and the WRNP, seeing that affine functions of the first Baire class satisfy the barycentric calculus (see [15], Chapter 12, Theorem, p. 100). From this point of view, we think it very important to deduce the equivalence between above two statements from that in the case where $X$ is separable. To this end, we first have:

Lemma 6 (Theorem 11 in [16]). Let $X$ be a separable Banach space and $K$ a weak*-compact convex subset of $X^{*}$. Then the following statements about $K$ are equivalent.

(a) The set K has the WRNP.

(b) The set $K$ has the scalar point of continuity property.

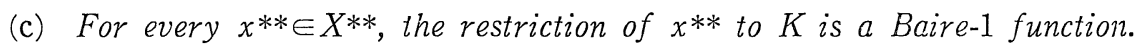

Proof. From our point of view, we give a proof of Lemma 6. The equivalence between the statements (b) and (c) follows from the Baire characterization theorem on the first Baire class, since $K$ is a compact metrizable space. Also the fact that (c) implies (a) follows from Theorem in [15] stated above and our Proposition 1. Finally, to see that (a) implies (b), suppose that (b) fails. Then, by the remark stated above, there exist a non-empty compact subset $S$ of $K$, real numbers $r$ and $\delta$ with $\delta>0$ and a bounded sequence $\left\{x_{n}\right\}_{n \geq 1}$ in $X$ such that putting $A_{n}=\left\{x^{*} \in S:\left(x^{*}, x_{n}\right) \geqq r+\delta\right\}$ and $B_{n}=\left\{x^{*} \in S:\left(x^{*}, x_{n}\right) \leqq r\right\}$ for all $n$, then $\bigcap_{n=1}^{\infty} \varepsilon_{n} A_{n} \neq \dot{\phi}$ for all $\left\{\varepsilon_{n}\right\}_{n \geqq 1}$ with $\varepsilon_{n}= \pm 1$. This means that a sequence $\left\{x_{n}\right\}_{n \geqq 1}$ has no point-wise convergent subsequence on $K$. So (a) fails by Proposition 4. Thus the proof is completed.

By the same argument as in Proposition 2 in [23] we have: 
Lemma 7. Let $K$ be a weak*-compact convex subset of $X^{*}$. If the set $K$ has the scalar point of continuity property, then so does $j *(K)$ for every closed subspace $Y$ of $X$, where $j$ is the inclusion map: $Y \rightarrow X$.

By Lemmas 6, 7 and Corollary 3, we have the following Proposition 5 concerning the equivalence between the statements (e) and $(\mathrm{g})$ in Theorem $\mathrm{E}$.

Proposition 5. Let $K$ be a weak*-compact convex subset of $X^{*}$. Then the set $K$ has the WRNP if and only if $K$ has the scalar point of continuity property.

Proof. Only if part can be proved by the same argument as in Lemma 6 . If part can be proved as follows. In virtue of Corollary 3 and Lemma 6, it suffices for us to show that $j *(K)$ has the scalar point of continuity property for every closed separable subspace $Y$ of $X$. But this is guaranteed by Lemma 7. So the proof is completed.

Thus we have studied the properties of weak*-compact convex sets and proved Theorem E from our standpoint to deal with a weak*-compact convex set itself and to clarify its structure directly (that is, in a process independent of Theorem A) by means of Theorems I, II and III. Consequently, setting $K=B_{X^{*}}$ in Propositions 1, 4 and 5, Rosenthal's theorem on Banach spaces containing a copy of $l_{1}$ yields :

Corollary 4 (cf. Theorem A and the remark after Theorem 2 in [21]). The following statements about $X$ are equivalent.

(a) Every $x^{* *} \in X^{* *}$ is universally measurable on $B_{X^{*}}$.

(b) Every $x^{* *} \in X^{* *}$ satisfies the barycentric calculus on $B_{X^{*}}$.

(c) The set $B_{X^{*}}$ is a set of complete continuity.

(d) The set $B_{X^{*}}$ is a set of complete continuity with respect to $([0,1], \Lambda, \lambda)$.

(e) The space $X^{*}$ has the WRNP.

(f) The space $X^{*}$ has the WRNP with respect to $([0,1], \Lambda, \lambda)$.

(g) The set $B_{X^{*}}$ has the scalar point of continuity property.

(h) The space $X$ does not contain a copy of $l_{1}$.

\section{References}

[1] Bourgain, J., Fremlin D.H. and Talagrand, M., Pointwise compact sets of Baire measurable functions, Amer. J, Math., 100 (1978), 845-886.

[2] Bourgin, R.D., Geometric aspects of convex sets with the Radon-Nikodym property, Lecture Notes in Math., 993, Springer, 1983.

[3] Davis, W. J., Figiel, T., Johnson, W.B. and Pelczynski, A., Factoring weakly compact operators, J. Funct. Anal., 17 (1974), 311-327.

[4] Diestel. J., Sequences and series in Banach spaces, Graduate Texts in Math., 92, Springer, 1984.

[5] Edgar, G. A., Measurable weak sections, Illinois J. Math., 20 (1976), 630-646. 
[6] Fremlin, D.H. and Talagrand, M., A decomposition theorem for additive set functions, with applications to Pettis integrals and ergodic means, Mati. Z., 168 (1979), 117-142.

[7] Haydon, R., Some more characterizations of Banach spaces containing $l_{1}$, Math. Proc. Cambridge Phil. Soc., 80 (1976), 269-276.

[8] Ionescu Tulcea, A. and Ionescu Tulcea C., Topics in the theory of lifting. Ergebnisse Math., 48, Springer, 1969.

[9] Janicka, L., Some measure theoretical characterization of Banach spaces not containing $l_{1}$, Bull. Acad. Polon. Sci., 27 (1979). 561-565.

[10] Matsuda, M., On Sierpinski's function and its applications to vector measures, Math. Japon., 28 (1983), 549-560.

[11] Musial, K., The weak Radon-Nikodym property in Banach spaces, Studia Math., 64 (1978), 151-174.

[12] Musial, K. and Ryll-Nardzewski, C., Liftings of vector measures and their applications to RNP and WRNP, Lecture Notes in Math., Springer, 645 (1978), 162-171.

[13] Odell, E. and Rosenthal, H.P., A double dual characterization of separable Banach spaces containing $l_{1}$, Israel J. Math., 20 (1975), 375-384.

[14] Pelczynski, A., On Banach spaces containing $L_{1}\left({ }^{\imath} \ell\right)$, Studia Math., 30 (1968), 231246.

[15] Phelps, R.R., Lectures on Choquet's theorem, Mathematical Studies, 7, Van Nostrand, 1966.

[16] Riddle, L.H., Saab, E. and Uhr, Jr, J.J., Sets with the weak Radon-Nikodym property in dual Banach spaces, Indiana Univ. Math. J., 32 (1983), 527-541.

[17] Riddle, L.H. and Uhr, Jr, J. J., Martingales and the fine line between Asplund spaces and spaces not containing a copy of $l_{1}$, Lecture Notes in Math., Springer, 939 (1981), 145-156.

[18] Rosenthal, H.P., A characterization of Banach spaces not containing $l_{1}$, Proc. Nat. Acad. Sci., 71 (1974), 2411-2413.

[19] , Some recent discoveries in the isomorphic theory of Banach spaces, Bull. Amer. Math. Soc., 84 (1978), 803-831.

[20] Saab, E., A characterization of $w^{*}$-compact convex sets having the Radon-Nikodym property, Bull. Sci. Math., (2) 104 (1980), 79-88.

[21] - On Dunford-Pettis operators that are Pettis-representable, Proc. Amer. Math. Soc., 85 (1982), 363-365.

[22] - Some characterizations of weak Radon-Nikodym sets, Proc. Amer. Math. Soc., 86 (1982), 307-311.

[23] Saab, E. and Saab, P., A dual geometric characterization of Banach spaces not containing $l_{1}$, Pacif. J. Math., 105 (1983), 415-425.

[24] Schwartz, L., Radon measures on arbitrary topological spaces and cylindrical measures, Tata institute of fundamental Reserch, Studies in Math., 6, Oxford University Press, 1973. 
